\title{
Effect of cartilage thickness mismatch in osteochondral grafting from knee to talus on articular contact pressures: A finite element analysis
}

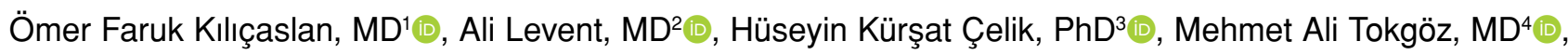 \\ Özkan Köse, MD'ㄹ, Allan E. W. Rennie, $\mathrm{PhD}^{5}$ (1) \\ ${ }^{1}$ Department of Orthopedics and Traumatology, HSU, Antalya Training and Research Hospital, Antalya, Turkey \\ ${ }^{2}$ Department of Orthopedics and Traumatology, HSU, Şanlıurfa Mehmet Akif Inan Training and Research Hospital, Şanlıurfa, Turkey \\ ${ }^{3}$ Department of Agricultural Machinery and Technology Engineering, Akdeniz University, Antalya, Turkey \\ 4Department of Orthopedics and Traumatology, HSU, Ankara Keçiören Training and Research Hospital, Ankara, Turkey \\ ${ }^{5}$ Department of Engineering, Lancaster University, Lancaster, United Kingdom
}

Osteochondral lesion of the talus (OLT) is a common disorder that causes chronic ankle pain, swelling, mechanical symptoms, and functional limitation. ${ }^{[1]}$ Small or medium-sized lesions usually benefit from arthroscopic procedures, such as debridement and microfracture; however, in large $\left(>150 \mathrm{~mm}^{2}\right)$, cystic and high-grade lesions, the long-term outcomes have been shown to worsen with unacceptable failure rates with arthroscopic microfractures. ${ }^{[2,3]}$ Therefore, cartilage restorative procedures, including autologous osteochondral grafting, autologous chondrocyte implantation (ACI), autologous matrix-induced

Received: March 08, 2021

Accepted: April 26, 2021

Published online: June 11, 2021

Correspondence: Özkan Köse, MD. Sağlık Bilimleri Üniversitesi, Antalya Eğitim ve Araştırma Hastanesi Ortopedi ve Travmatoloji Kliniği, 07100 Muratpaşa, Antalya, Türkiye.

E-mail: drozkankose@hotmail.com

Doi: $10.52312 / j d r s .2021 .41$

Citation: Kılıçaslan ÖF, Levent A, Celik HK, Tokgöz MA, Köse Ö, Rennie AEW. Effect of cartilage thickness mismatch in osteochondral grafting from knee to talus on articular contact pressures: A finite element analysis. Jt Dis Relat Surg 2021;32(2):355-362.

(O2021 All right reserved by the Turkish Joint Diseases Foundation

This is an open access article under the terms of the Creative Commons Attribution-NonCommercial License, which permits use, distribution and reproduction in any medium, provided the original work is properly cited and is not used for commercial purposes (http://creativecommons.org/licenses/by-nc/4.0/).

\section{ABSTRACT}

Objectives: The aim of this study was to investigate the effect of cartilage thickness mismatch on tibiotalar articular contact pressure in osteochondral grafting from femoral condyles to medial talar dome using a finite element analysis (FEA).

Materials and methods: Flush-implanted osteochondral grafting was performed on the talar centromedial aspect of the dome using osteochondral plugs with two different cartilage thicknesses. One of the plugs had an equal cartilage thickness with the recipient talar cartilage and the second plug had a thicker cartilage representing a plug harvested from the knee. The ankle joint was loaded during a single-leg stance phase of gait. Tibiotalar contact pressure, frictional stress, equivalent stress (von Mises values), and deformation were analyzed.

Results: In both osteochondral grafting simulations, tibiotalar contact pressure, frictional stress, equivalent stress (von Mises values) on both tibial and talar cartilage surfaces were restored to near-normal values.

Conclusion: Cartilage thickness mismatch does not significantly change the tibiotalar contact biomechanics, when the graft is inserted flush with the talar cartilage surface.

Keywords: Cartilage, mosaicplasty, osteochondral grafting, osteochondral lesions, talus; tibiotalar contact pressure.

chondrogenesis (AMIC), particulated juvenile cartilage allograft, or osteochondral allograft transplantation, have been advocated for large OLTs. ${ }^{[4-6]}$

Among these treatment options, autologous osteochondral grafting has been shown to be safe and effective with successful clinical results in primary or revision cases. ${ }^{[5]}$ This technique has various advantages. First, it is a single-stage procedure and 
can be performed with simple surgical equipment. Second, and most importantly, the hyaline cartilage is directly transferred to the defective area, independent of the healing capacity of the recipient site. Third, both the cartilage and the underlying subchondral and cancellous bone are simultaneously substituted, which is a critical advantage in the presence of subchondral cyst formation and bony defect. $^{[1,4,5]}$

Finite element analysis (FEA) can help us to understand biomechanics better, select optimal choices, and make clinical decisions. It can be used as a powerful non-invasive tool to evaluate the biomechanical properties of prior and novel techniques and to simulate surgical procedures for clinical cases. It can be also used as an alternative to studies that expensive, require specialized devices for its implementation, and ethically sensitive animals or cadavers. ${ }^{[7]}$ The FEA can potentially mimic cartilage morphology using mesh elements (tetrahedral, hexahedral), material properties (elastic, hyperelastic, poroelastic, composite), physiological loads by applying loading conditions (static, dynamic), and constitutive stress-strain equations (linear, porous-elastic, biphasic). ${ }^{[8]}$

Previous studies have shown that elevated, recessed, or angled osteochondral plugs significantly deteriorate the joint contact pressures. ${ }^{[9-11]}$ Besides, it is well-known that articular cartilage thickness is subject to variations between joints. Furthermore, it is not even uniform within the same joint. ${ }^{[12-15]}$ Therefore, even with flush placement of osteochondral plugs with a thicker cartilage, it may result in a subchondral bone surface mismatch (Figure 1). ${ }^{[16]}$
Theoretically, cartilage thickness mismatch may adversely affect the joint contact pressures. However, cartilage thickness mismatch between donor and recipient sites has not been well-studied previously and still remains unclear. In light of the existing data, in the present study, we aimed to investigate the cartilage thickness mismatch on the tibiotalar contact pressure from knee to talus autologous osteochondral grafting using FEA.

\section{MATERIALS AND METHODS}

The FEA was carried out in linear static loading conditions and assumptions of a homogeneous isotropic linear elastic material model. The non-linear contact between components of the model was also defined. Osteochondral autografting was performed on the centromedial aspect of the talar dome using osteochondral plugs with two different cartilage thicknesses. Since OLTs are most frequently seen in the centromedial region, ${ }^{[17]}$ this area was chosen for simulations. One of the plugs had an equal cartilage thickness with the recipient talar cartilage and the second plug had a thicker cartilage representing a plug harvested from the knee. An intact tibiotalar joint and an empty socket were also simulated as control operations.

\section{Modeling of the ankle joint and osteochondral grafting}

To construct a realistic digital model, an intact ankle joint was modeled based on computed tomography (CT) data of a healthy male individual who was $184 \mathrm{~cm}$ in height and $98 \mathrm{~kg}$ in weight. The CT examination (Siemens go.Up, Siemens Healthineers
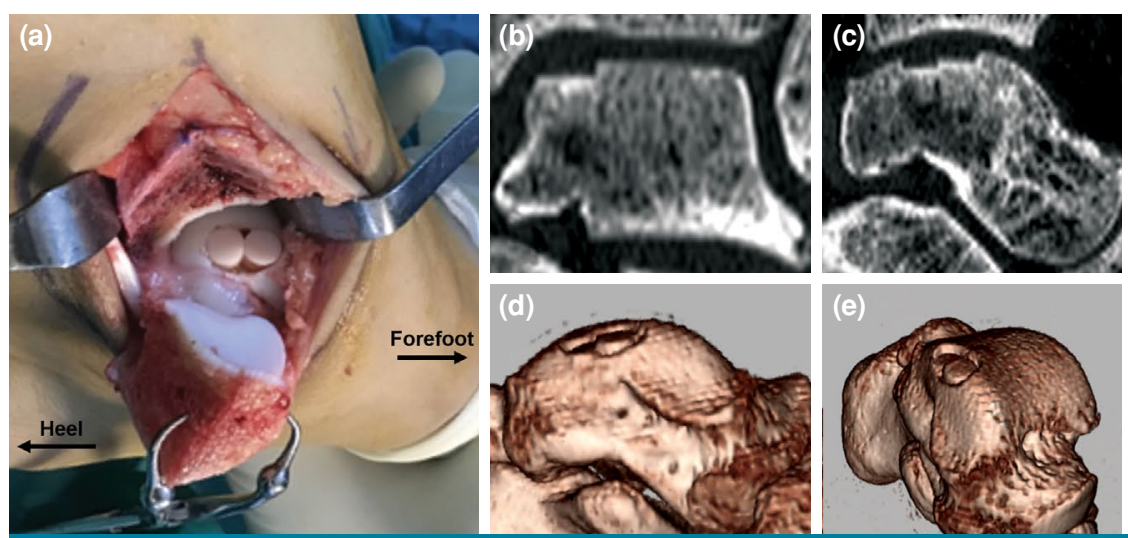

(f)

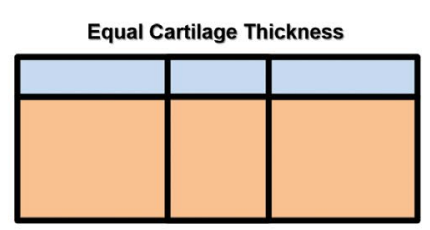

Cartilage Thickness Mismatch

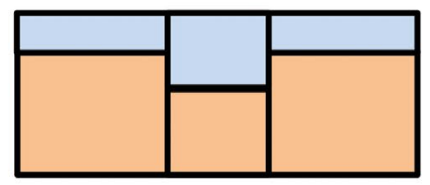

FIGURE 1. (a) Intraoperative appearance of two osteochondral grafts harvested from lateral femoral condyles. Note flush placement of plugs with talar articular surface. (b) Coronal and (c) sagittal CT of talus showing subchondral surface mismatch. (d, e) Three-dimensional CT images showing the subchondral surface irregularity. (f) Schematic drawing showing differences between equal cartilage thickness and mismatch.

CT: Computed tomography. 
(a)

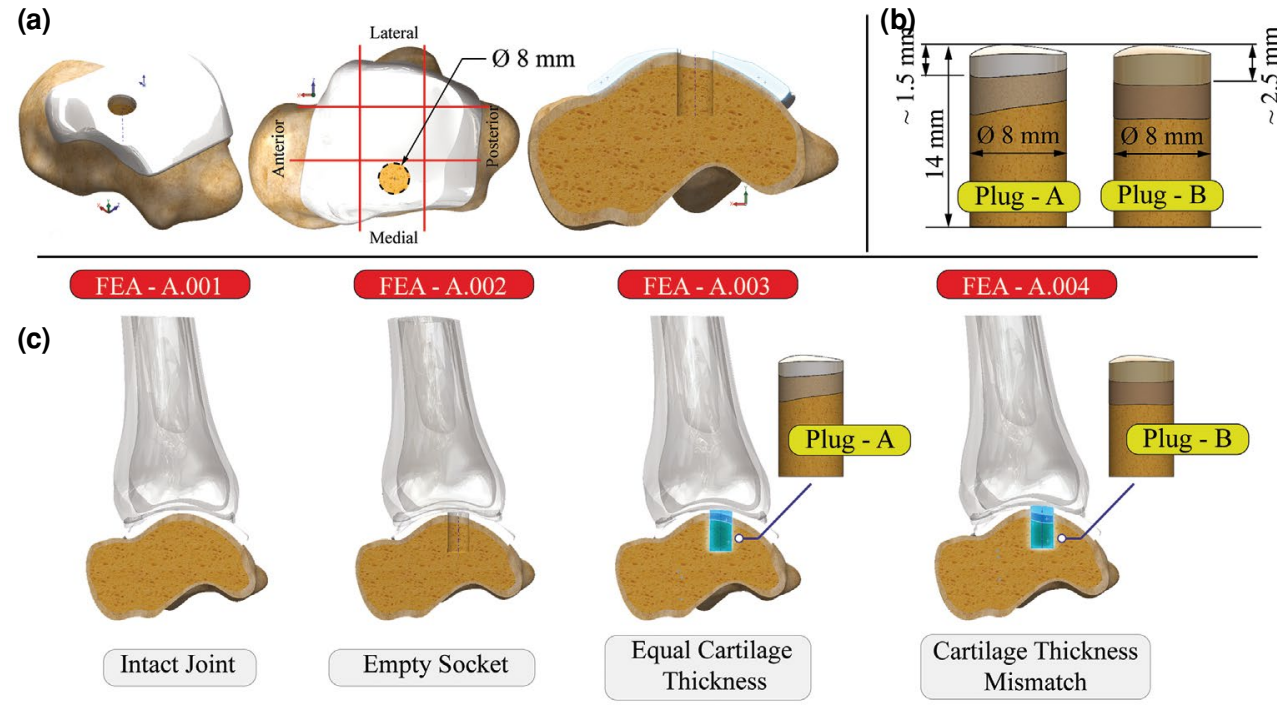

FIGURE 2. (a) Localization of plugs on talar articular surface. (b) Thickness of cartilage in plug A and B was $1.5 \mathrm{~mm}$ and $2.5 \mathrm{~mm}$, respectively. Total height of plug was $14 \mathrm{~mm}$. (c) Schematic presentation of FEA simulation scenarios.

FEA: Finite element analysis.

AG, Munich, Germany) was performed in the supine position with a slice distance of $0.7 \mathrm{~mm}$ from $80 \mathrm{~mm}$ above the ankle joint down to the heel $(130 \mathrm{KV}, 42 \mathrm{~mA}$, 334 slices). The $\mathrm{CT}$ examination revealed no osseous lesions and deformity. A written informed consent for the confidential use of the imaging files received from the participant. The study was conducted in accordance with the principles of the Declaration of
Helsinki. The 3D Slicer version 4.10.2 (3D Slicer, BWH, MA, USA), Meshmixer version 3.5 (Autodesk, CA, USA), SolidWorks 2020 (Dassault Systèmes SolidWorks Corp., MA, USA), and ANSYS ${ }^{\circledR}$ workbench version 17.0 (ANSYS, Inc., PA, USA) were applied to model and simulate the FEA scenarios, respectively.

In the solid modeling, cortical bone, trabecular bone, and cartilage were separately created and

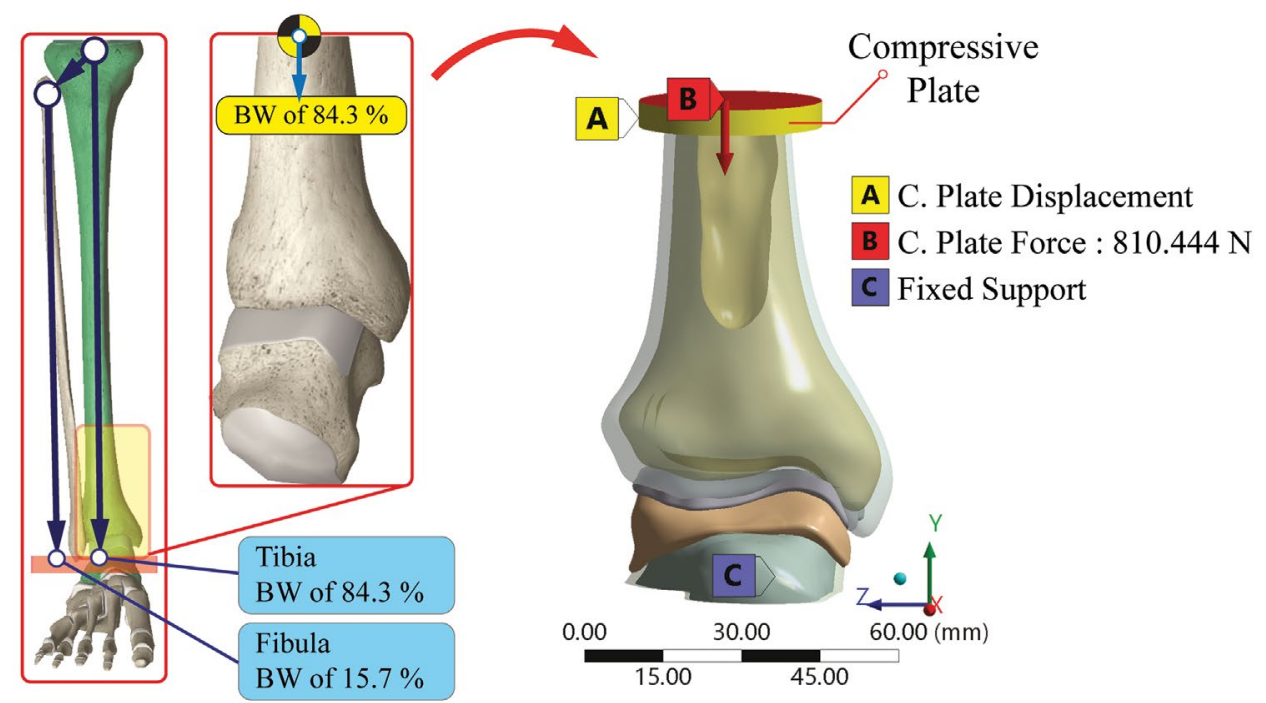

FIGURE 3. Boundary conditions. Tibial column carries $84.3 \%$ of total body weight (BW). Since the participant was $98 \mathrm{~kg}$, the model was loaded with $810.4 \mathrm{~N}(\mathrm{~B})$ using a compressive plate (A) on talus (C). Loading was applied as axial compression. 


\begin{tabular}{|c|c|c|c|c|}
\hline Material propertie & $\begin{array}{l}\text { ed in the } \\
\text { line }\end{array}$ & $\begin{array}{c}\text { TABLE I } \\
\text { A set-up in accc } \\
\text { elastic material }\end{array}$ & $\begin{array}{l}\text { lance with the hon } \\
\text { odel }\end{array}$ & nous isotro \\
\hline \multirow[b]{2}{*}{ Parameters } & \multirow[b]{2}{*}{ Unit } & \multicolumn{3}{|c|}{ Model components } \\
\hline & & Cortical bone & Trabecular bone & Cartilage \\
\hline Elastic modulus & $(\mathrm{MPa})$ & $19100^{[26,27]}$ & $1000.61^{[28,29]}$ & $1230^{[31]}$ \\
\hline Poisson's ratio & $(-)$ & $0.3^{[30]}$ & $0.3^{[30]}$ & $0.42^{[31]}$ \\
\hline Density & $\left(\mathrm{kg} \mathrm{m}^{-3}\right)$ & $1980^{[30]}$ & $830^{[32]}$ & $431^{[30]}$ \\
\hline
\end{tabular}

assembled. Cartilage layer was modeled based on CT scan images and full surface contact visualization for bone-to-cartilage and cartilage-to-cartilage was ensured to obtain a non-uniform realistic cartilage thickness distribution. Average thickness values of the articular cartilage between the tibia and talus varied between 0.52 and $2.85 \mathrm{~mm}$ in the modeling operation. The cartilage contact surface area between the tibia and talus was measured as $1120.59 \mathrm{~mm}^{2}$. These thickness values for articular cartilage were compatible within an acceptable range with the literature reported for articular cartilage. ${ }^{[14,15,18-21]}$ The solid modeling details and localization of the grafting are illustrated in Figure 2.

An 8-mm diameter osteochondral plug was inserted perpendicular to the talar articular surface. While the first plug had the same cartilage thickness $(1.5 \mathrm{~mm})$ as the talar articular cartilage, the second osteochondral plug had a 2.5-mm cartilage thickness to mimic an osteochondral plug taken from the knee. In previous cadaveric studies, the lateral femoral trochlea's cartilage thickness was reported to be $2.5 \mathrm{~mm}$ on average. ${ }^{[12,15,16,22]}$ Both the plugs and the socket were $14 \mathrm{~mm}$ in length and were inserted press-fit similar to the ideal osteochondral grafting technique.

\section{Boundary conditions and material properties}

The ankle joint model was loaded in a neutral position to replicate a single-leg weight-bearing posture. The participant's body weight $(98 \mathrm{~kg})$ was used as the reference value to calculate the loading magnitude. A previous biomechanical study reported that the tibial column carried $84.3 \%$ of the body weight, and the rest was carried by the fibular column. ${ }^{[23]}$ The loading magnitude carried by the tibial column was calculated as $810.44 \mathrm{~N}$ $(98 \mathrm{~kg} \times 9.81 \mathrm{~m} \mathrm{~s}-2 \times 0.843)$ (embedded gravity effect), and this was assigned in the simulations. Boundary conditions set-up in the simulation scenarios are illustrated in Figure 3. The contact definitions between components were included as
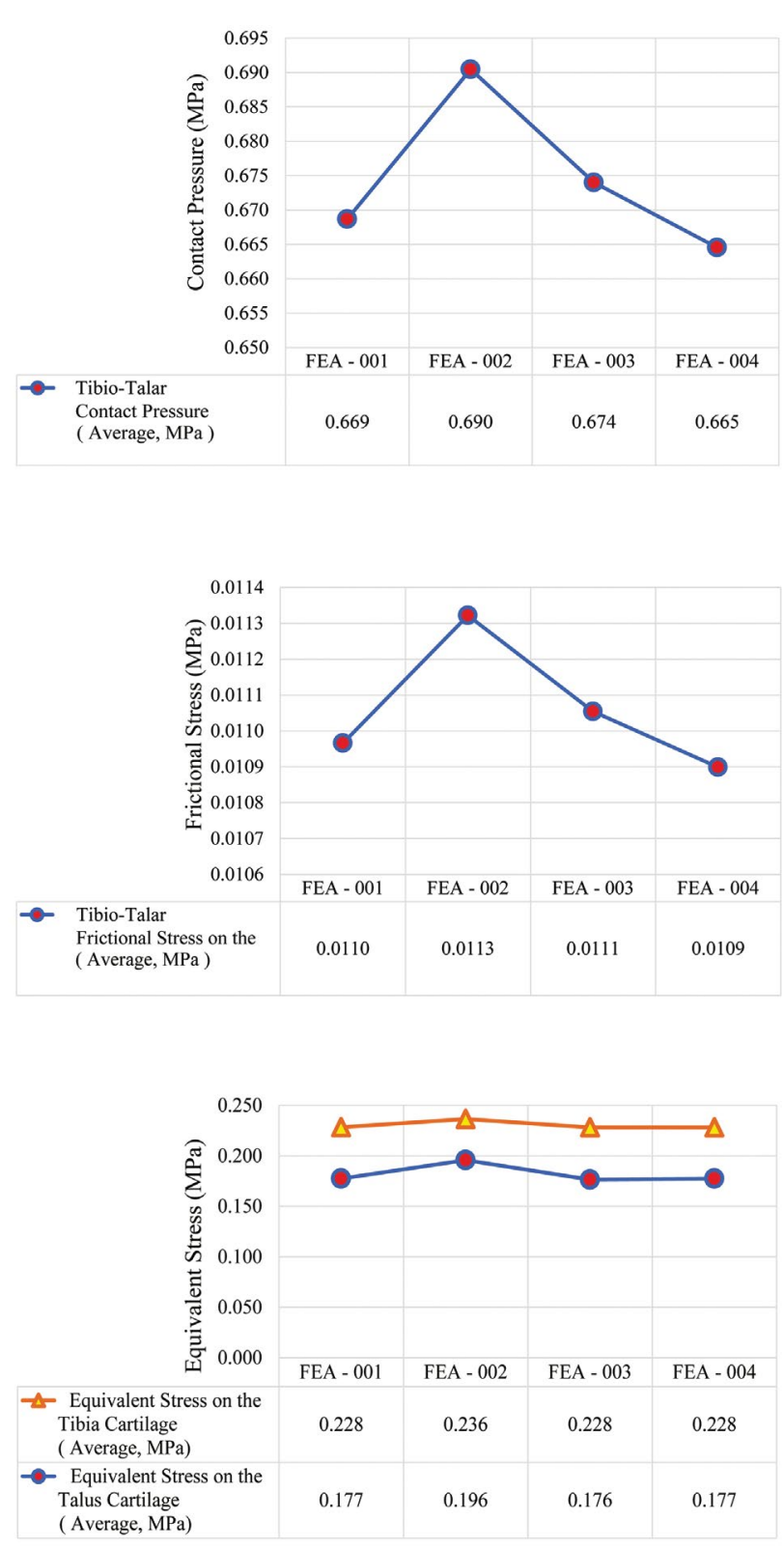

FIGURE 4. Graph showing contact pressure, frictional stress, and equivalent stress on articular cartilages in each tested FEA scenario.

FEA: Finite element analysis. 
the frictional contact (non-linear contact) between the osteochondral plug and the socket and tibiotalar articular cartilage surfaces. Furthermore, bonded contact definitions were defined between cortical and trabecular bone and subcortical bone (frictional coefficient: 0.46 ) and the articular cartilage (frictional coefficient: 0.0164$).^{[24,25]}$

The material properties defined in the FEA were collected based on the previous data in the literature. The material properties for cortical, trabecular, and articular cartilage were separately assigned under consideration of isotropic homogenous linear elastic material model assumptions (Table I). ${ }^{[26-32]}$

\section{Mesh structure and quality verification}

The mesh structure of the finite element (FE) has been shown to influence FEA outputs directly, and minimum criteria for model selection, proper recognition of parameters, and verification have been defined to achieve a precise performance and output. ${ }^{[33]}$ To validate the current FE model for the predefined FEA scenarios, both mesh density (sensitivity) measurement and skewness metric (mesh quality) controls were performed. The mesh sensitivity analysis findings suggested a minimum element size of $1 \mathrm{~mm}$ for the tibial cortical and trabecular bone. Besides, for intact tibiotalar joint and osteochondral grafting models, average skewness values of 0.217 and 0.235 were obtained, respectively. The $1-\mathrm{mm}$ element size and skewness values resulted in an excellent mesh quality for the FE models (total number of elements: 628570, total number of nodes: 926132). As a result, all simulation scenarios were performed using an identical curvature meshing strategy. The Dell Precision M4800 Series (Intel Core ${ }^{\mathrm{TM}}$ i7 4910MQ CPU@ 2.90 GHz, NVIDIA Quadro K2100M-2GB, and Physical Memory: 32 GB) mobile workstation was employed as the solving platform.

\section{Tibiotalar joint biomechanical evaluations}

Visual outputs, numerical values of the equivalent stress (von Mises values), and total tibiotalar structure deformation distributions on the components, tibiotalar articular cartilage contact pressure, frictional stress, and contact pressure between all fragments were extracted from the simulation results and presented with graphs. No statistical analysis was performed, as the measurements obtained in FEA were absolute values without any variation.

\section{RESULTS}

The average contact pressure between tibial and talar cartilage was $0.669 \mathrm{MPa}$ in the intact ankle joint. In the empty socket simulation, joint contact pressure increased to $0.690 \mathrm{MPa}$. The osteochondral grafting with the same cartilage thickness increased the contact pressure by $0.74 \%$, relative to the intact ankle joint, and reached $0.674 \mathrm{MPa}$. A plug with a

\begin{tabular}{|c|c|c|c|c|c|}
\hline Summary & $\begin{array}{l}\text { ABLE II } \\
\text { umerical FE }\end{array}$ & utputs & & & \\
\hline & & & FEA st & y code & \\
\hline FEA output & Value & FEA-001 & FEA-002 & FEA-003 & FEA-004 \\
\hline Contact pressure on the surface between tibia and talus & Maximum & 1.677 & 1.708 & 1.653 & 1.683 \\
\hline cartilages (MPa) & Mean & 0.669 & 0.690 & 0.674 & 0.665 \\
\hline Frictional stress on the surface between tibia and talus & Maximum & 0.0275 & 0.0280 & 0.0271 & 0.0276 \\
\hline cartilages (MPa) & Mean & 0.0110 & 0.0113 & 0.0111 & 0.0109 \\
\hline 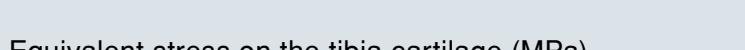 & Maximum & 1.305 & 1.297 & 1.216 & 1.226 \\
\hline Equivalent stress on tne tiola cartilage (IVIra) & Mean & 0.228 & 0.236 & 0.228 & 0.228 \\
\hline 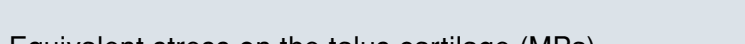 & Maximum & 1.004 & 1.017 & 0.973 & 0.976 \\
\hline Equivalent stress on the talus cartllage (MPa) & Mean & 0.177 & 0.196 & 0.176 & 0.177 \\
\hline Equivalent stress on the plug (MPa) & Maximum & - & - & 8.006 & 7.343 \\
\hline Deformation on the plug $(\mathrm{mm})$ & Maximum & - & - & 0.103 & 0.117 \\
\hline Deformation on the plug (Y-direction) (mm) & Maximum & - & - & 0.103 & 0.116 \\
\hline Deformation of the total body loading ( $\mathrm{mm})$ & Maximum & 0.312 & 0.306 & 0.313 & 0.314 \\
\hline Deformation of the total body loading (Y-direction) (mm) & Maximum & 0.301 & 0.296 & 0.302 & 0.303 \\
\hline
\end{tabular}




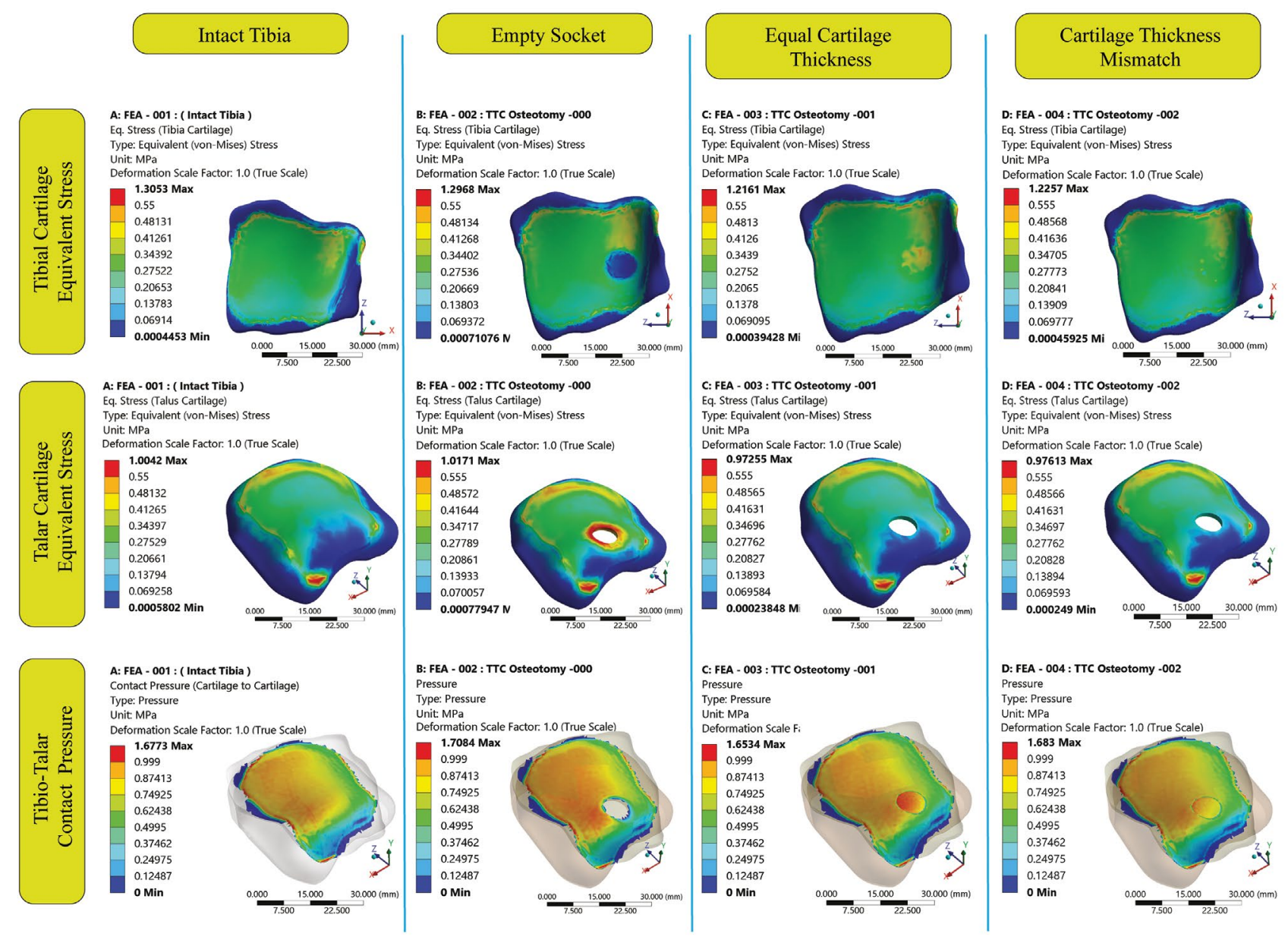

FIGURE 5. Summary of visual outputs.

thicker cartilage decreased the contact pressure to $0.665 \mathrm{MPa}(0.59 \%$, compared to the intact ankle joint) (Figure 4).

The average frictional stress between tibial and talar cartilage was similar in all tested scenarios. The summary of the results is presented in Table II. Under defined boundary conditions, no permanent deformation or damage was detected on any of the analyzed components (cortical bone, trabecular bone, and cartilage). The maximum equivalent (von Mises) stress values on each component were far less than their yield stress values (Figure 5). In case of a cartilage defect (FEA-002), the equivalent stress concentrated around the periphery of the defect on talar cartilage. On the contrary, reciprocal tibial cartilage was unloaded. In both osteochondral grafting scenarios, the equivalent stress values on tibial and talar cartilage decreased to near-normal values.

\section{DISCUSSION}

In the current study, we investigated the biomechanical effects of cartilage thickness mismatch between donor and recipient area in autologous osteochondral grafting from knee to talus. Our study results showed that osteochondral plugs with a relatively thicker cartilage layer did not cause a significant change in contact pressure, frictional stress, equivalent stress, and deformation in medially located OLT treatment, despite the presence of subchondral stepping. Based on these findings, we can speculate that osteochondral plugs of different thicknesses may be used for autologous osteochondral grafting. However, it should be noted that the osteochondral plug was inserted flush to the joint.

The importance of the flush placement of osteochondral plugs has been also shown in previous cadaveric studies. Latt et al. ${ }^{[9]}$ compared joint contact pressures in flush, recessed $(5 \mathrm{~mm}$ and $10 \mathrm{~mm}$ ), and elevated $(5 \mathrm{~mm}$ and $10 \mathrm{~mm})$ osteochondral plugs 
using 10 human cadaveric ankles and reported that flush graft placement restored joint contact pressure near-intact values, whereas either recessed or elevated graft placement increased the joint contact pressures. Similarly, in an FEA study conducted by Li et al., ${ }^{[34]}$ cartilage defects deeper than $1 \mathrm{~mm}$ had a significant effect on the ankle joint contact pressure. However, there was no linear correlation between defect thickness and contact pressure over $1 \mathrm{~mm}$. In addition to flush placement, the angle of graft insertion is also an important variable that affects the joint contact pressures. In an animal cadaveric model, Koch et al. ${ }^{[35]}$ demonstrated that the elevated edge of the angled grafts led to a significant increase in joint contact pressures. Based on their results, the authors recommended both perpendicular graft harvesting from the recipient site and perpendicular insertion to the donor site to obtain optimal surface congruency.

Despite a consensus on how the grafts should be placed concerning height and angle in the relevant literature, only one previous study investigated the effect of cartilage thickness mismatch. In a cadaveric study, Fansa et al. ${ }^{[10]}$ created an osteochondral defect at the centromedial aspect of the talar dome, and an autologous osteochondral graft from the ipsilateral knee was attempted to place flush to the defect site. The autologous osteochondral graft was able to be placed $1.0 \mathrm{~mm}$ below and $0.4 \mathrm{~mm}$ above the surrounding tissue with varying discrepancies, and persistent deficits in contact mechanics were found, even if optimal surface congruence was provided. The authors proposed that differences in material properties (elastic modulus, stiffness) and cartilage thickness might be responsible for this discrepancy. Our findings differ from the aforementioned study. As the thickness of the cartilage increased, the pressure absorption capacity increased; thus, it did not change the joint contact pressure significantly.

Furthermore, the talar and femoral cartilage have been shown to be different in respect to morphological, biological, and biomechanical properties. Treppo et al. ${ }^{[36]}$ found that the talar cartilage was denser with a higher sulfated glycosaminoglycan content, lower water content, higher equilibrium modulus, and dynamic stiffness, and lower hydraulic permeability. Henak et al. ${ }^{[3]}$ showed that femoral cartilage was softer than the talar cartilage, particularly near the articular surface, and had a lower friction coefficient. The aforementioned authors suggested that these differences could cause failure following osteochondral grafting from the knee to the talus and suggested that local grafts taken from the talus itself would be a more suitable option, as they could mimic the surface geometry and the biology and biomechanical properties. Although the most frequently used donor site for harvesting osteochondral plugs is the knee joint, local grafts may be harvested from the ipsilateral anterior part of the medial or lateral facets of the talus as an alternative. ${ }^{[38]}$ This approach may decrease the donor site morbidity at the knee joint, reported in around $15 \%$ of the patients. In addition, Georgiannos et al. ${ }^{[39]}$ reported that talar osteochondral plugs had a similar cartilage thickness, as well as similar biological and biomechanical properties. However, topographic studies demonstrated that cartilage thickness was different between different joints and also within the same joint, which is basically related to the different exposure to load. ${ }^{[12,14]}$

Nonetheless, there are some limitations to this study. Although FEA is a valuable complementary method to understand the mechanical behavior of biological materials, it is vulnerable to various errors that may occur at each stage of the computerized analysis. ${ }^{[40]}$ The simplified modeling used in this analysis may not fully reflect the complex anatomy of the ankle, including soft tissues, ligaments, and muscles. Several assumptions in defining material properties and boundary conditions were also used. Due to the FEA-specific constraints, the outputs might not have replicated real-life exactly; however, even the approximate results can provide significant information.

In conclusion, osteochondral grafts taken from the knee can be used in the repair of OLT without changing the joint biomechanics. However, harvesting the grafts perpendicular to the donor area and flush and perpendicular placement to the recipient area are two critical factors to obtain near-normal joint contact biomechanics. Further clinical studies are required to gain a better understanding of how these differences affect the functional outcomes and prognosis.

\section{Declaration of conflicting interests}

The authors declared no conflicts of interest with respect to the authorship and/or publication of this article.

\section{Funding}

The authors received no financial support for the research and/or authorship of this article.

\section{REFERENCES}

1. Looze CA, Capo J, Ryan MK, Begly JP, Chapman C, Swanson D, et al. Evaluation and management of osteochondral lesions of the talus. Cartilage 2017;8:19-30.

2. van Bergen CJ, Kox LS, Maas M, Sierevelt IN, Kerkhoffs GM, van Dijk CN. Arthroscopic treatment of osteochondral defects of the talus: Outcomes at eight to twenty years of follow-up. J Bone Joint Surg [Am] 2013;95:519-25. 
3. Choi WJ, Park KK, Kim BS, Lee JW. Osteochondral lesion of the talus: Is there a critical defect size for poor outcome? Am J Sports Med 2009;37:1974-80.

4. Savage-Elliott I, Ross KA, Smyth NA, Murawski CD, Kennedy JG. Osteochondral lesions of the talus: A current concepts review and evidence-based treatment paradigm. Foot Ankle Spec 2014;7:414-22.

5. Shimozono Y, Hurley ET, Myerson CL, Kennedy JG. Good clinical and functional outcomes at mid-term following autologous osteochondral transplantation for osteochondral lesions of the talus. Knee Surg Sports Traumatol Arthrosc 2018;26:3055-62.

6. Korucu İH, Kekeç AF, Arslan A, Oltulu P, Korucu EN, Özer M. Regenerative effects of hyperbaric oxygen therapy and plateletrich plasma on the osteochondral defects of rats. Jt Dis Relat Surg 2020;31:260-6

7. Ye Y, You W, Zhu W, Cui J, Chen K, Wang D. The applications of finite element analysis in proximal humeral fractures. Comput Math Methods Med 2017;2017:4879836.

8. Hassan CR, Qin YX, Komatsu DE, Uddin SMZ. Utilization of finite element analysis for articular cartilage tissue engineering. Materials (Basel) 2019;12:3331.

9. Latt LD, Glisson RR, Montijo HE, Usuelli FG, Easley ME. Effect of graft height mismatch on contact pressures with osteochondral grafting of the talus. Am J Sports Med 2011;39:2662-9.

10. Fansa AM, Murawski CD, Imhauser CW, Nguyen JT, Kennedy JG. Autologous osteochondral transplantation of the talus partially restores contact mechanics of the ankle joint. Am J Sports Med 2011;39:2457-65.

11. Kock NB, Smolders JM, van Susante JL, Buma P, van Kampen A, Verdonschot $\mathrm{N}$. A cadaveric analysis of contact stress restoration after osteochondral transplantation of a cylindrical cartilage defect. Knee Surg Sports Traumatol Arthrosc 2008;16:461-8.

12. Ahmad CS, Cohen ZA, Levine WN, Ateshian GA, Mow VC. Biomechanical and topographic considerations for autologous osteochondral grafting in the knee. Am J Sports Med 2001;29:201-6.

13. Buettner O, Leumann A, Lehner R, Dell-Kuster S, Rosenthal R, Mueller-Gerbl M, et al. Histomorphometric, CT arthrographic, and biomechanical mapping of the human ankle. Foot Ankle Int 2013;34:1025-34.

14. Millington SA, Grabner M, Wozelka R, Anderson DD, Hurwitz SR, Crandall JR. Quantification of ankle articular cartilage topography and thickness using a high resolution stereophotography system. Osteoarthritis Cartilage 2007;15:205-11.

15. Shepherd DE, Seedhom BB. Thickness of human articular cartilage in joints of the lower limb. Ann Rheum Dis 1999;58:27-34.

16. Urita A, Cvetanovich GL, Madden BT, Verma NN, Inoue $\mathrm{N}$, Cole BJ, et al. Topographic matching of osteochondral allograft transplantation using lateral femoral condyle for the treatment of medial femoral condyle lesions: A computer-simulated model study. Arthroscopy 2018;34:3033-42.

17. Elias I, Zoga AC, Morrison WB, Besser MP, Schweitzer ME, Raikin SM. Osteochondral lesions of the talus: Localization and morphologic data from 424 patients using a novel anatomical grid scheme. Foot Ankle Int 2007;28:154-61.

18. Sugimoto K, Takakura Y, Tohno Y, Kumai T, Kawate K, Kadono K. Cartilage thickness of the talar dome. Arthroscopy 2005;21:401-4.

19. Wan L, de Asla RJ, Rubash HE, Li G. Determination of in-vivo articular cartilage contact areas of human talocrural joint under weightbearing conditions. Osteoarthritis Cartilage 2006;14:1294-301.

20. Maier J, Black M, Bonaretti S, Bier B, Eskofier B, Choi JH, et al. Comparison of different approaches for measuring tibial cartilage thickness. J Integr Bioinform 2017;14:20170015.

21. Ding M, Dalstra M, Danielsen CC, Kabel J, Hvid I, Linde F. Age variations in the properties of human tibial trabecular bone. J Bone Joint Surg [Br] 1997;79:995-1002.
22. Terukina M, Fujioka H, Yoshiya S, Kurosaka M, Makino T, Matsui $\mathrm{N}$, et al. Analysis of the thickness and curvature of articular cartilage of the femoral condyle. Arthroscopy 2003;19:969-73.

23. Wang Q, Whittle M, Cunningham J, Kenwright J. Fibula and its ligaments in load transmission and ankle joint stability. Clin Orthop Relat Res 1996;(330):261-70.

24. Hayden LR, Escaro S, Wilhite DR, Hanson RR, Jackson RL. A comparison of friction measurements of intact articular cartilage in contact with cartilage, glass, and metal. JBBBE 2019;41:23-35.

25. Eberle S, Gerber C, von Oldenburg G, Högel F, Augat P. A biomechanical evaluation of orthopaedic implants for hip fractures by finite element analysis and in-vitro tests. Proc Inst Mech Eng H 2010;224:1141-52.

26. Dong XN, Acuna RL, Luo Q, Wang X. Orientation dependence of progressive post-yield behavior of human cortical bone in compression. J Biomech 2012;45:2829-34.

27. Wang $X$, Nyman JS, Dong $X$, Leng $H$, Reyes $M$, editors. Fundamental biomechanics in bone tissue engineering synthesis lectures on tissue engineering. 1st ed. Williston: Morgan \& Claypool; 2010.

28. Kim SH, Chang SH, Jung HJ. The finite element analysis of a fractured tibia applied by composite bone plates considering contact conditions and time-varying properties of curing tissues. Composite Structures 2010;92:2109-18.

29. Klekiel T. Będziński R. Finite element analysis of large deformation of articular cartilage in upper ankle joint of occupant in military vehicles during explosion. Arch Metall Mater 2015;60:2115-21.

30 Alonso-Rasgado T, Jimenez-Cruz D, Karski M. 3-D computer modelling of malunited posterior malleolar fractures: Effect of fragment size and offset on ankle stability, contact pressure and pattern. J Foot Ankle Res 2017;10:13.

31. Zhu ZJ, Zhu Y, Liu JF, Wang YP, Chen G, Xu XY. Posterolateral ankle ligament injuries affect ankle stability: a finite element study. BMC Musculoskelet Disord 2016;17:96.

32. Novitskaya E, Zin C, Chang N, Cory E, Chen P, D'Lima D, et al. Creep of trabecular bone from the human proximal tibia. Mater Sci Eng C Mater Biol Appl 2014;40:219-27.

33. Viceconti M, Olsen S, Nolte LP, Burton K. Extracting clinically relevant data from finite element simulations. Clin Biomech (Bristol, Avon) 2005;20:451-4.

34. Li J, Wei Y, Wei M. Finite element analysis of the effect of talar osteochondral defects of different depths on ankle joint stability. Med Sci Monit 2020;26:e921823.

35. Koh JL, Kowalski A, Lautenschlager E. The effect of angled osteochondral grafting on contact pressure: a biomechanical study. Am J Sports Med 2006;34:116-9.

36. Treppo S, Koepp H, Quan EC, Cole AA, Kuettner KE, Grodzinsky AJ. Comparison of biomechanical and biochemical properties of cartilage from human knee and ankle pairs. J Orthop Res 2000;18:739-48.

37. Henak CR, Ross KA, Bonnevie ED, Fortier LA, Cohen I, Kennedy $\mathrm{JG}$, et al. Human talar and femoral cartilage have distinct mechanical properties near the articular surface. J Biomech 2016;49:3320-7.

38. Kreuz PC, Steinwachs M, Erggelet C, Lahm A, Henle P, Niemeyer P. Mosaicplasty with autogenous talar autograft for osteochondral lesions of the talus after failed primary arthroscopic management: a prospective study with a 4 -year follow-up. Am J Sports Med 2006;34:55-63.

39. Georgiannos D, Bisbinas I, Badekas A. Osteochondral transplantation of autologous graft for the treatment of osteochondral lesions of talus: 5- to 7-year follow-up. Knee Surg Sports Traumatol Arthrosc 2016;24:3722-9.

40. Cheung JT, Zhang M. A 3-dimensional finite element model of the human foot and ankle for insole design. Arch Phys Med Rehabil 2005;86:353-8. 